\title{
Near infrared photometric and optical spectroscopic study of 22 low mass star clusters embedded in nebulae ${ }^{\star}$
}

\author{
J. B. Soares ${ }^{1,2}$, E. Bica ${ }^{1}$, A. V. Ahumada ${ }^{3}$, and J. J. Clariá ${ }^{3}$ \\ ${ }^{1}$ Universidade Federal do Rio Grande do Sul, IF, CP 15051, Porto Alegre 91501-970, RS, Brazil \\ e-mail: jules@if.ufrgs.br \\ 2 LATO-DCET-UESC, Rodovia Ilhéus-Itabuna, km 16, Ilhéus 45662-000, BA, Brazil \\ 3 Observatorio Astronómico de Córdoba, Universidad Nacional de Córdoba, Laprida 854, 5000 Córdoba, Argentina
}

Received 4 May 2007 / Accepted 3 October 2007

\section{ABSTRACT}

\begin{abstract}
Aims. Among the star clusters in the Galaxy, those embedded in nebulae represent the youngest group, which has only recently been explored. The analysis of a sample of 22 candidate embedded stellar systems in reflection nebulae and/or HII environments is presented.

Methods. We employed optical spectroscopic observations of stars in the directions of the clusters carried out at CASLEO (Argentina) together with near infrared photometry from the 2MASS catalogue. Our analysis is based on source surface density, colour-colour diagrams and on theoretical pre-main sequence isochrones. We take into account the field star contamination by carrying out a statistical subtraction.

Results. The studied objects have the characteristics of low mass systems. We derive their fundamental parameters. Most of the cluster ages are younger than 2 Myr. The studied embedded stellar systems in reflection nebulae and/or HII region complexes do not have stars of spectral types earlier than B. The total stellar masses locked in the clusters are in the range 20-220 $M_{\odot}$. They are found to be gravitationally unstable and are expected to dissolve in a timescale of a few Myr.
\end{abstract}

Key words. Galaxy: open clusters and associations: general

\section{Introduction}

Apart from globular and open clusters in the Galaxy, a new class of star clusters has been detected in recent decades thanks to the development of infrared detectors (see Lada \& Lada 2003, for a review). They are the clusters embedded in nebulae, which are in general stellar systems newly formed in giant molecular clouds and associated with emission and/or reflection nebulae. Bica et al. (2003a) provided a catalogue of 276 embedded clusters and stellar groups. The 2MASS database (Skrutskie et al. 2006, and references therein) allowed the discovery of many new embedded clusters (e.g. Ivanov et al. 2002; Dutra et al. 2003; Bica et al. 2003b).

Total masses of embedded clusters within 2 kpc occur mostly in the range of 20 to $1000 M_{\odot}$; however, the efforts to study them have been mainly concentrated on those with intermediate and higher total masses (Lada \& Lada 2003). On the other hand, lower mass embedded clusters are interesting because they may contain non-ionizing stars as their most massive members, thus producing particular interactions with the interstellar medium (Soares \& Bica 2002, 2003; Soares 2006). Optical stars were spectroscopically observed in Soares et al. (2005) aiming at studying the more massive possible members.

The goal of the present work is to carry out a photometric analysis in the near infrared of the stellar content of a sample of 22 low mass cluster candidates (Dutra et al. 2003) from the

^ Based on observations made at Complejo Astronómico El Leoncito, which is operated under agreement between the Consejo Nacional de Investigaciones Científicas y Técnicas de la República Argentina and the National Universities of La Plata, Córdoba and San Juan, Argentina.
2MASS catalogue. We also determine spectral types for possible optical members by using spectroscopic observations carried out at Complejo Astronómico El Leoncito (CASLEO, Argentina). In Sect. 2 we present the selected sample of low mass cluster candidates. In Sect. 3 we provide the photometric and spectroscopic data and in Sect. 4 we describe the methods used. The cluster properties are analysed in Sect. 5 and the concluding remarks are presented in Sect. 6.

\section{The embedded star cluster sample}

The selected sample of 22 embedded clusters and stellar groups (Table 1) has been taken from Dutra et al. (2003). This sample is not too densely populated and they are all embedded in nebulae (see Sect. 5.2 and Table 1).

Due to the limited resolution of the 2MASS catalogue, we excluded the objects with known distances of the nebulae larger than $2.4 \mathrm{kpc}$ as well as those presenting high stellar density. We illustrate in Fig. 1, DBSB 002 in the HII region Gum 5 and DBSB 011 in the nebula Bran 22C. This figure provides typical IR images for two objects of the current sample.

Table 1 shows the physical and structural parameters for the objects in the selected sample. The columns indicate: (1) object identification; (2) and (3) equatorial coordinates (J2000); (4) related nebula; (5) nebula environment - HII region (HII) or reflection nebula (RN); (6) known distance of the nebula; (7) derived cluster angular radius in arcmin; (8) richness index (Sect. 4); (9) $A_{J}$ mean absorption; (10) $K_{\mathrm{s}}$ excess fraction $\left(f_{K_{\mathrm{s}}}\right)$; (11) total mass. 
Table 1. Structural and physical parameters for the clusters and related nebulae.

\begin{tabular}{|c|c|c|c|c|c|c|c|c|c|c|}
\hline Cluster & $\alpha(\mathrm{J} 2000)$ & $\delta(\mathrm{J} 2000)$ & Nebulae & environment & $d_{\odot}[\mathrm{kpc}]$ & $r_{a}{ }^{a}$ & $I c^{b}$ & $A_{J}^{c}$ & $f_{K_{\mathrm{s}}}$ & $M\left[M_{\odot}\right]$ \\
\hline DBSB 002 & 071012 & -182621 & Gum 5 & HII & - & 1.0 & 28 & $1.70 \pm 0.08$ & 0.19 & - \\
\hline DBSB $011^{\dagger}$ & 072416 & -243801 & Bran 22C & - & - & 2.0 & 84 & $1.18 \pm 0.07$ & 0.33 & - \\
\hline DBSB 014 & 073746 & -325029 & Bran 58 & - & - & 1.6 & 38 & $1.97 \pm 0.10$ & 0.29 & - \\
\hline DBSB 030 & 090154 & -444332 & Bran 221 & - & - & 0.6 & 9 & $2.23 \pm 0.16$ & - & - \\
\hline DBSB 031 & 085230 & -484557 & vdBH-RN 22a & $\mathrm{RN}$ & 1.7 & 1.4 & 36 & $1.91 \pm 0.12$ & 0.30 & 67 \\
\hline DBSB 035 & 091511 & -472832 & Bran 242 & - & - & 2.0 & 52 & $1.07 \pm 0.12$ & 0.25 & - \\
\hline DBSB 039 & 092425 & -530808 & Bran 262 & - & - & 0.6 & 10 & $0.74 \pm 0.14$ & - & - \\
\hline DBSB 040 & 100122 & -574310 & Bran 280 & RN & - & 1.6 & 41 & $1.23 \pm 0.11$ & 0.31 & - \\
\hline DBSB 041 & 100128 & -574349 & Bran 280 & RN & - & 1.2 & 28 & $1.63 \pm 0.15$ & 0.45 & - \\
\hline DBSB 042 & 100733 & -573330 & Bran 288, RCW 46 & HII & - & 0.6 & 11 & $1.06 \pm 0.19$ & - & - \\
\hline DBSB 049 & 105108 & -542045 & Brana 322B & - & - & 2.0 & 57 & $0.99 \pm 0.10$ & 0.21 & - \\
\hline DBSB 064 & 112441 & -585629 & vdBH-RN 48 & $\mathrm{RN}$ & 1.5 & 0.6 & 19 & $0.86 \pm 0.10$ & 0.11 & 35 \\
\hline DBSB 069 & 113354 & -631646 & Bran 66, RCW 62 & HII & 1.7 & 1.0 & 20 & $0.68 \pm 0.10$ & 0.18 & 31 \\
\hline DBSB 070 & 114836 & -621716 & Bran 373 & HII & 1.7 & 1.0 & 16 & $0.86 \pm 0.19$ & 0.11 & 31 \\
\hline DBSB 081 & 125840 & -662105 & vdBH-RN 58 & RN & 1.7 & 1.0 & 22 & $0.45 \pm 0.11$ & - & - \\
\hline DBSB 087 & 133247 & -602654 & ESO 132EN 12 & HII & - & 0.6 & 14 & $2.18 \pm 0.45$ & - & - \\
\hline DBSB $088^{\ddagger}$ & 141940 & -612520 & RCW 85 & HII & 1.2 & 1.2 & 14 & $2.73 \pm 0.53$ & 0.22 & 20 \\
\hline DBSB 104 & 170402 & -510455 & vdBH-RN 81 & RN & - & 2.0 & 35 & $0.48 \pm 0.04$ & 0.11 & - \\
\hline DBSB 109 & 164711 & -411628 & vdBH-RN 73a & RN & 1.9 & 1.6 & 42 & $1.28 \pm 0.12$ & 0.07 & 120 \\
\hline DBSB 114 & 165910 & -401205 & G $345.3+1.5$ & HII & 1.9 & 1.2 & 65 & $1.72 \pm 0.08$ & 0.18 & 219 \\
\hline DBSB 121 & 171701 & -362210 & RCW 126 & HII & 1.4 & 1.6 & 42 & $2.41 \pm 0.25$ & 0.50 & 78 \\
\hline DBSB 123 & 171715 & -362018 & RCW 126 & HII & 1.4 & 1.6 & 54 & $2.61 \pm 0.21$ & 0.20 & 130 \\
\hline
\end{tabular}

${ }^{a}$ Cluster limiting angular radius (arcmin); ${ }^{b}$ richness indicator index; ${ }^{c} A_{J}$ mean absorption.

$\dagger$ Same as IBP 6 (Ivanov et al. 2002).

¥ In Dutra et al. (2003) the declination is incorrect but the related nebula is correct. This cluster is the same as GLIMPSE-50 (Mercer et al. 2005).
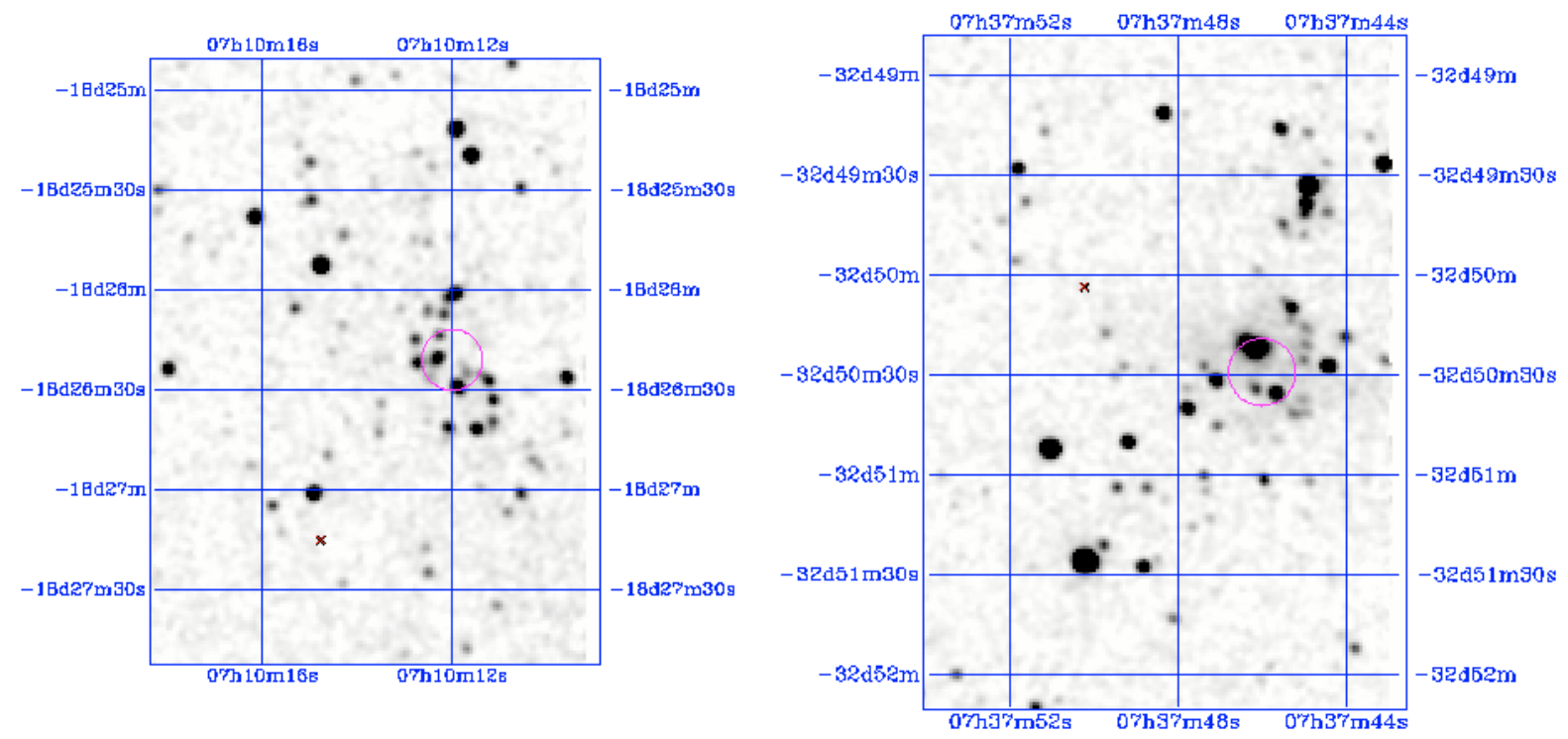

Fig. 1. 2MASS $K_{\mathrm{s}}$ band images of DBSB 002 (left) and DBSB 011 (right) showing clustering. The small open circle indicates the object's central part.

Object identification and further information about the nebulae shown in Table 1 come from several studies: RCW (Rodgers et al. 1960), Gum (Gum 1955), Bran (Brand et al. 1986), ESO (Lauberts 1982) and G (Caswell \& Haynes 1987; Kuchar e Clark 1997). The columns dealing with the cluster parameters are discussed in Sect. 5.

\section{Photometric and spectroscopic data}

The photometric data were taken from the Two Micron All Sky Survey (2MASS). We employed the Gator tool for Point Source
Catalog extractions, as given in the $2 \mathrm{MASS}$ site ${ }^{1}$. An extraction table gives for each star, the $J, H$ and $K_{\mathrm{s}}$ magnitudes, the three corresponding colours $\left(J-H, H-K_{\mathrm{s}}\right.$ and $\left.J-K_{\mathrm{S}}\right)$, the respective errors and the $\mathbf{J} 2000.0$ coordinates. The $2 \mathrm{MASS}$ photometric errors in the present regions for each magnitude are basically the same as those presented in Soares \& Bica (2002) for the regions of NGC 2327 and BRC 27. The errors become large (0.1 mag or larger) for magnitudes fainter than $J=15.7, H=15$ or $K_{\mathrm{s}}=14$. For comparison purposes we made extractions up to a radius $r=$ $5^{\prime}$ around the object center.

${ }^{1}$ http://www.ipac.caltech.edu/2mass 


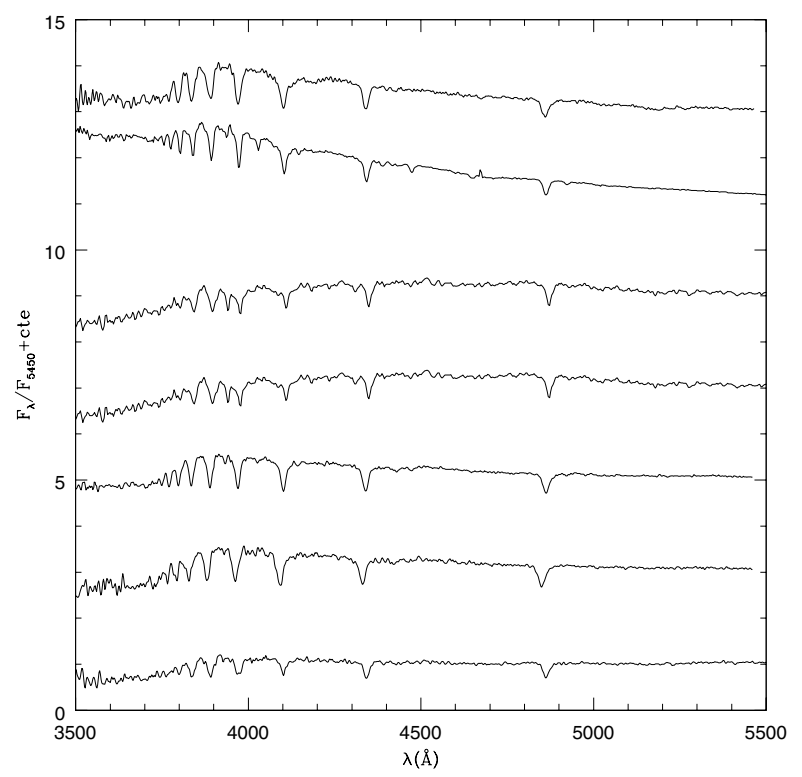

Fig. 2. Observed spectra of the stars in Table 2. From top to bottom: DBSB031_1, DBSB039_1, DBSB039_2, DBSB042_1, DBSB049_1, DBSB049_2 and DBSB069_1. Spectra are normalised to $F_{\lambda}=1$ at $\lambda=5450 \AA$. Constants have been added to the spectra, except for the bottom one.

The spectroscopic observations were carried out with the Jorge Sahade $2.15 \mathrm{~m}$ telescope at CASLEO (Argentina) over 11 nights between October 2005 and April 2006. In all observing runs, we employed a CCD camera containing a Tektronix chip of $1024 \times 1024$ pixels attached to a REOSC spectrograph (simple mode), the size of each pixel being $24 \mu \times 24 \mu$. The total field along the slit was $4.7^{\prime}$, with the slit oriented in the east-west direction. We obtained spectra ranging from $3500 \AA$ to $5600 \AA$, using a grating of 300 lines $/ \mathrm{mm}$. The average dispersion was $140 \AA / \mathrm{mm}$. Three exposures of 20 min were generally taken for each object. Standard stars LTT 1020, LTT 2415, LTT 2511, LTT 3864, LTT 7987 and LTT 9239 (Stone \& Baldwin 1983) were used for calibration of the observed spectra. For instrumental calibration purposes, frames of $\mathrm{Cu}-\mathrm{Ar}-\mathrm{Ne}$ comparison lamps were taken between and after the object observations, as well as bias, dome, twilight sky and tungsten lamp flat-fields. The spectra were reduced using the IRAF package. The description of reduction procedures can be seen in Ahumada et al. (2000). The observed spectra are shown in Figs. 2 and 3. The continuum distributions indicate various amounts of reddening.

\section{Methods of analysis}

We obtained spectral types for the brightest star(s) in each object by means of the template matching method, namely, by achieving the best possible match between the continuum and lines of the analysed star spectrum and the stars in the spectral library of Silva \& Cornell (1992).

The analysis method is the same as that used by Ahumada et al. (2001). For the sake of illustrating the method, we show in Fig. 4 the observed spectrum of the star DBSB 049_3, its dereddened spectrum and a B6V template spectrum. From such comparisons, we derived spectral types and reddening values (Table 2).

The source density profile is useful when studying the angular size of star clusters and their number of stellar sources. The angular radius for each star cluster is obtained by considering

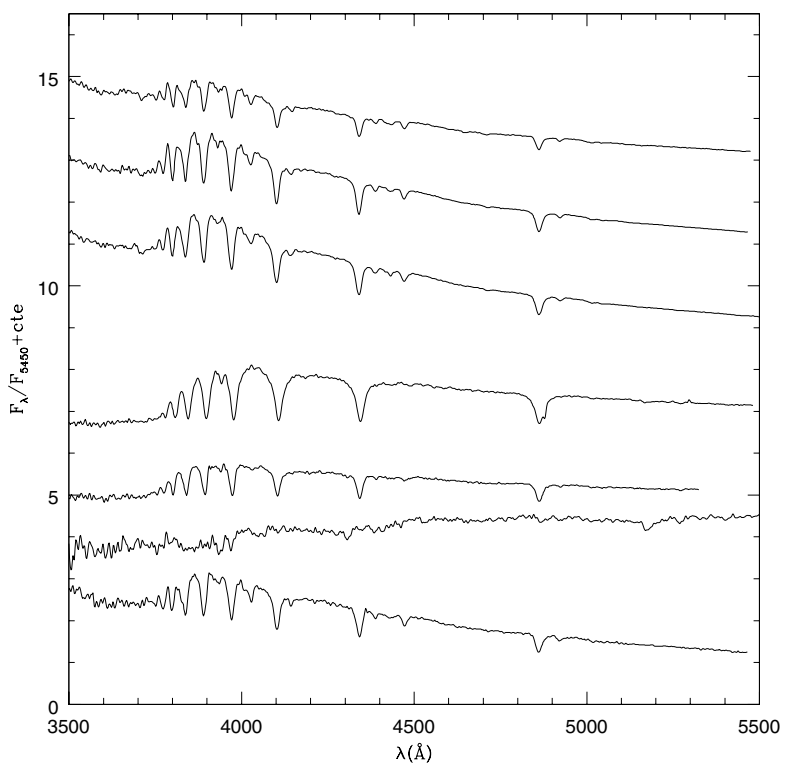

Fig. 3. Same as in Fig. 2 for the remaining stars. From top to bottom: DBSB069_2, DBSB070_1, DBSB081_1, DBSB088_1, DBSB104_1, DBSB104_2 and DBSB109_1.

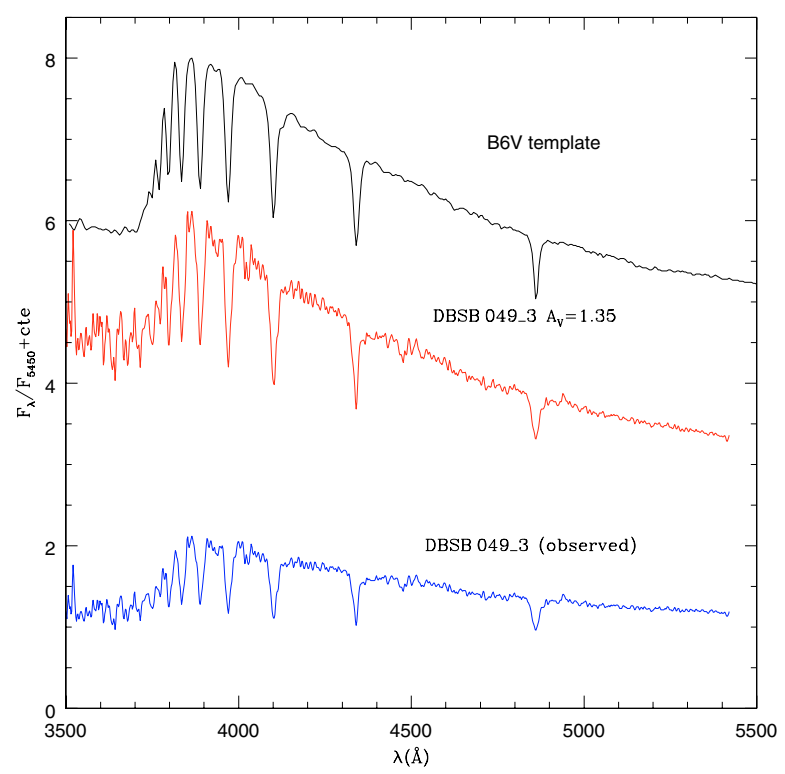

Fig. 4. Observed spectrum of the DBSB 049_3 star, the same spectrum corrected of reddening and the B6V template spectrum (Silva \& Cornell 1992). The spectra are normalised at $\lambda=5450 \AA$ and shifted by arbitrary constants for comparison purposes, except for the bottom one.

the mean value of the source density profile of the surrounding stellar field.

The number of star cluster members is estimated using the Ic index, i.e., considering the ratio between the number of sources in the direction of the star cluster and that of the surrounding stellar field (Testi et al. 1998). We extracted stars within $5^{\prime}$ of the assumed center of each object (Table 1), using the 2MASS photometry. By dividing this area into concentric $0.2^{\prime}$ wide rings, we calculated the number of stars confined within each ring per unit area. The Ic index does not take into account differential extinction. The young cluster regions are expected to present larger extinctions than those of their 


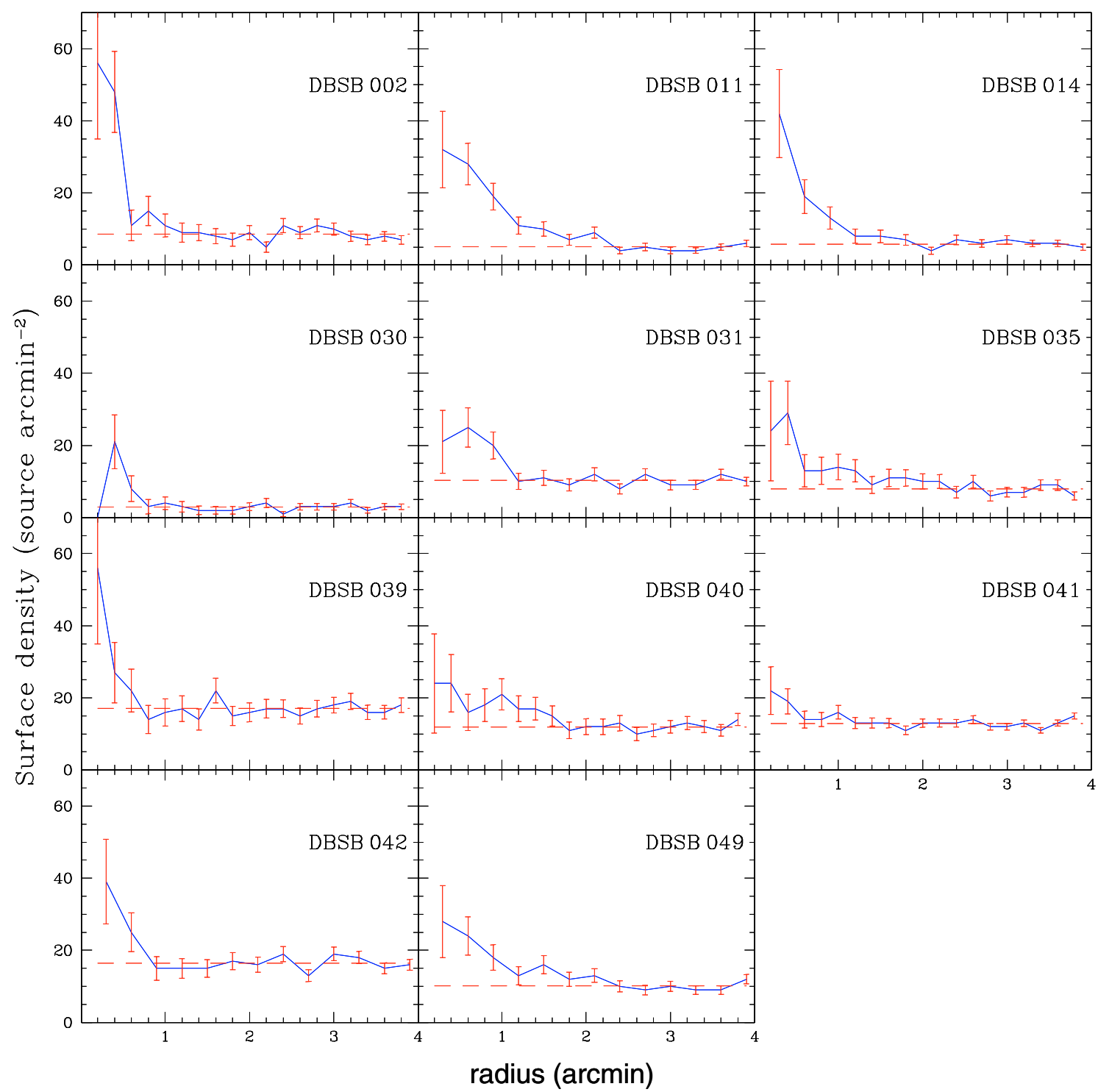

Fig. 5. Source surface density distributions for the studied embedded clusters or stellar groups. The dashed lines indicate the mean background surface density for each object. Poisson errors are indicated.

surroundings. Considering this expected effect, the Ic index could overestimate the observed field star contamination in the cluster regions, resulting in an underestimation of cluster members.

The stellar field was removed from each star cluster by means of a statistical method. The comparisons of the distribution of stars in the colour-colour diagram (2CD) for on-cluster and off-cluster stars are the essence of this statistical field subtraction method. This associates a membership probability $\left(P_{\text {jon }}\right)$ with each star in the on-cluster area. Details are given in Soares et al. (2005). The 2CD analysis allows us to derive the mean extinction of the embedded clusters, using reddening vectors and intrinsic colours as in Soares et al. (2005). In addition, the $2 \mathrm{CD}$ analysis provides information about the evolutionary stage of the cluster, through the fraction of stars with $K_{\mathrm{s}}$ band excess, which is characteristic of pre-main sequence (PMS) stars (Carpenter et al. 1993; Lada \& Adams 1992).

The estimated mass for each star cluster was obtained by means of a method similar to that of Lada \& Lada (2003). We adopted a universal initial mass function (IMF) for all the clusters, using the Trapezium cluster IMF derived by Muench et al. (2002) in a study of the luminosity function in the $K_{\mathrm{s}}$ band.

$$
\frac{\mathrm{d} N}{\mathrm{~d} m}=M^{\alpha}\left\{\begin{array}{l}
\alpha=-2.21, M>0.600 M_{\odot} \\
\alpha=-1.15,0.600 M_{\odot}<M<0.120 M_{\odot} \\
\alpha=-0.27,0.120 M_{\odot}<M<0.025 M_{\odot} \\
\alpha=-6.00,0.025 M_{\odot}<M<0.017 M_{\odot} .
\end{array}\right.
$$

The stellar mass of the faintest detected star in each cluster was obtained considering the detection limit, the distance and the mean extinction for each cluster. The derived mass for the clusters depends on their evolutionary stage. Therefore, isochrones of different ages were used in this analysis.

To derive the total mass for each cluster, we estimated the total number of stars first considering the number of detected stars and the IMF of the Trapezium cluster. We used the following relation between the total mass $\left(M_{\mathrm{T}}\right)$ and the total number of stars $\left(N_{\mathrm{T}}\right)$ derived for the Trapezium, $M_{\mathrm{T}} / N_{\mathrm{T}}=0.53$ (Muench et al. 2002).

The cluster age can be estimated by means of the $K_{\mathrm{s}}$ excess method (Soares et al. 2005, and references therein). In this 


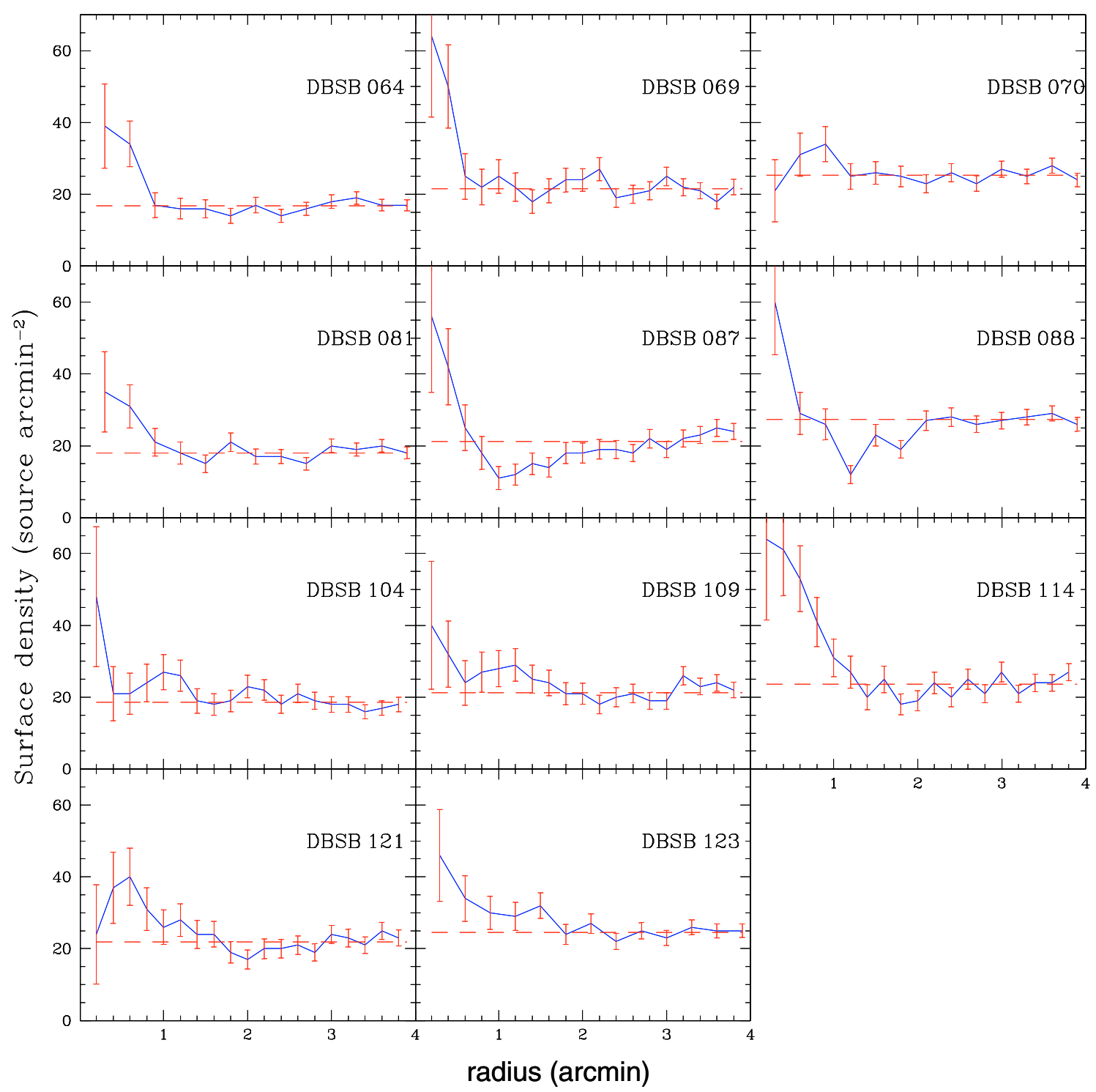

Fig. 6. Same as in Fig. 5 for the remaining objects.

method the essential information is given by those stars which present $K_{\mathrm{s}}$ excess and by the total number of cluster members. The $K_{\mathrm{s}}$ fractions for each cluster are given in Table 1 .

An estimate of the age for each cluster with known distance was obtained by means of a second method, which involves PMS evolutionary tracks and isochrones of D'Antona \& Mazzitelli (1997, 1998). The evolutionary tracks range from $0.07 \mathrm{Myr}$ to $100 \mathrm{Myr}$ for PMS stars with masses from $0.17 M_{\odot}$ to $3 M_{\odot}$. We adopted a deuterium abundance of $2 \times 10^{-5}$ and a metallicity of $Z=0.02$. We converted the theoretical plane into the observational one by using the bolometric corrections of Schmidt-Kaler (1982). For PMS stars younger than 0.3 Myr, we adopted this lower limit age. The age fitting was applied to probable member stars which were individually corrected for absorption (Sect. 4).

\section{Analysis}

\subsection{Individual stars}

Spectroscopic data were obtained for 10 out of the 22 clusters. The brightest stars in each cluster were observed. Table 2 shows
Table 2. Positions and spectroscopic results for the brightest stars in the nebulae direction.

\begin{tabular}{cccccc}
\hline \hline Star & $\alpha(\mathbf{J} 2000)$ & $\delta(\mathbf{J} 2000)$ & $J[\mathrm{mag}]$ & ST & $A_{\mathrm{V}}$ \\
\hline DBSB031_1 & 085229 & -484612 & 11.681 & B34V & 2.79 \\
DBSB039_1 & 092424 & -530759 & 11.909 & A7V & 0.96 \\
DBSB039_2 & 092425 & -530801 & 12.727 & A57V & 1.11 \\
DBSB042_1 & 100730 & -573259 & 7.424 & A89IV & 1.35 \\
DBSB049_1 & 105106 & -542042 & 9.574 & A57V & 0.60 \\
DBSB049_2 & 105109 & -542025 & 12.747 & B0V & 1.44 \\
DBSB049_3 & 105106 & -542051 & 12.554 & B6V & 1.35 \\
DBSB069_1 & 113354 & -631638 & 10.437 & B5V & 1.56 \\
DBSB069_2 & 113353 & -631652 & 11.358 & B34V & 0.81 \\
DBSB070_1 & 114837 & -621726 & 12.316 & G9K0V & 0.21 \\
DBSB081_1 & 125840 & -662218 & 11.070 & B6V & 1.74 \\
DBSB088_1 & 141937 & -612504 & 11.724 & A13V & 0.75 \\
DBSB104_1 & 170401 & -510501 & 8.163 & B34V & 0.30 \\
DBSB104_2 & 170359 & -510457 & 8.550 & B34V & 0.45 \\
DBSB109_1 & 164710 & -411631 & 9.526 & B12V & 1.17 \\
\hline
\end{tabular}

coordinates, $J$-magnitudes, spectral types and the resulting extinction values $A_{\mathrm{V}}$. 


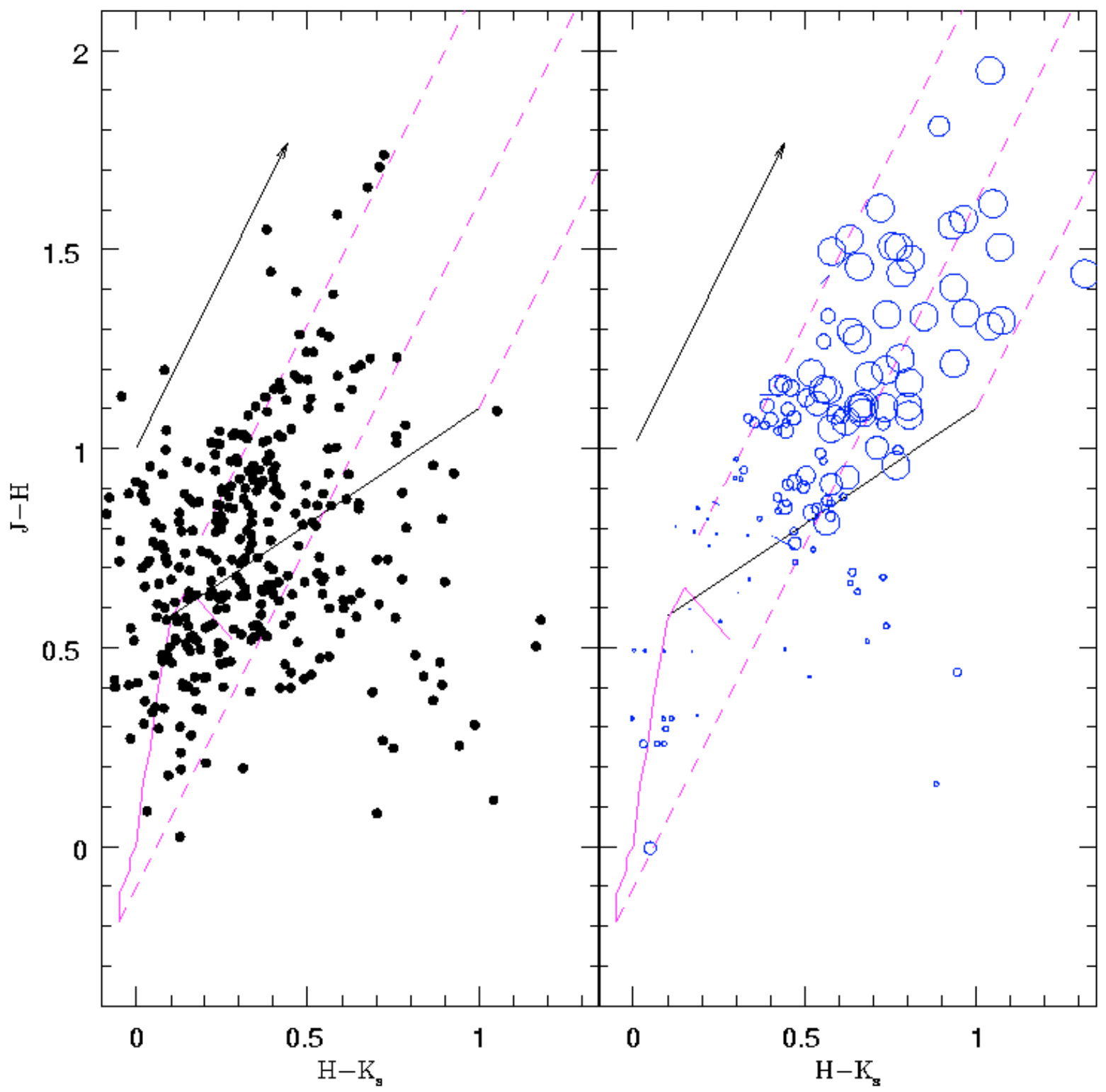

Fig. 7. Illustration of the statistical field star subtraction on the 2CD of DBSB 011. The left and right panels show on- and off-cluster stars respectively. Symbol sizes are proportional to the $P_{\text {jon }}$ membership probabilities. Reference lines as in Fig. 8.

The bright stars DBSB 042_1, DBSB 049_1 and DBSB 070_1 appear to be foreground stars, all of which affected by low extinction. The earlier spectral type and the absorption obtained for the other stars suggest that they are members of their associated cluster.

The distance moduli for these stars imply distances compatible with the values found for the related nebulae in the literature (Table 1). In the sample of clusters with available spectroscopic data, the DBSB 049 cluster presents the most massive member, a B0V star, according to the derived spectral type. Therefore, clusters in this sample seem to have been formed without the presence of a massive star earlier than B-type, including those clusters situated in an HII region environment.

The extinction derived from the spectroscopy is low if compared with typical mean extinctions for embedded clusters. However, these clusters exhibit a considerable differential reddening. This fact suggests that the dust column in the direction of the brightest star in the cluster is smaller on average than the dust columns of the rest of the stars. This is possibly due to stellar winds.

\subsection{Structure}

The relation between source surface density and angular radial distance for the clusters is shown in Figs. 5 and 6 . The vertical bars are Poissonian errors. The dashed line indicates the mean star field density, which is obtained by means of the stars contained in the outermost rings (radii from $2^{\prime}$ to $4^{\prime}$ ). The clusters DBSB 11, DBSB 14, DBSB 31, DBSB 42, DBSB 49, DBSB 64, DBSB 70, DBSB 81, DBSB 88 and DBSB 123 are shown in Figs. 5 and 6, with increased radial bin size $\left(0.3^{\prime}\right)$, to get a more reliable profile. The surface density profile (SDP) of sources presents a significant contrast for most of the star clusters in the sample, when it is compared with the SDP of the background. 


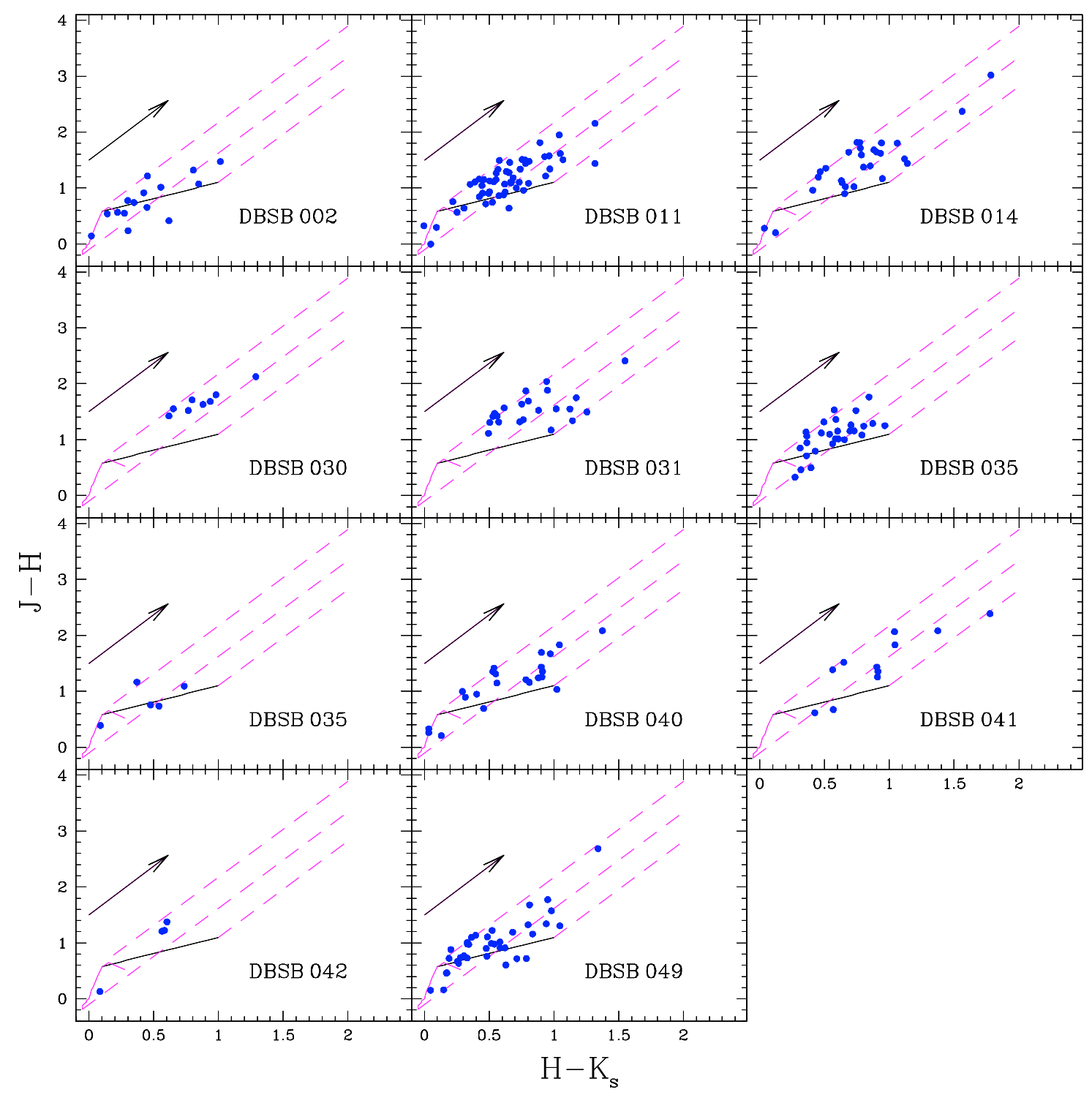

Fig. 8. $\left((J-H),\left(H-K_{\mathrm{s}}\right)\right)$ diagrams. The continuous curve represents the intrinsic distribution of spectral types and the continuous straight line represents the unreddened locus of T Tauri stars. The reddening vector indicates $A_{\mathrm{V}}=5$. Reddening lines for M 5 giants, O3 stars and T Tauri stars are shown as dashed lines. Faint sources with prohibitive errors were excluded. Each diagram is a realization of the statistical subtraction.

As expected, the larger the $I c$ index, the larger the contrast between star cluster and its surrounding stellar field in a SDP. For example, the DBSB 114 cluster turned out to have $I c=65$, according to its SDP (Table 1).

The angular radius is defined as the limiting radius for the cluster, where the SDP of the object becomes indistiguishable from its surrounding stellar field. The angular radii derived for the sample are in the range of $0.6^{\prime}$ to $2.0^{\prime}$ (Table 1), corresponding to a linear radius in the range of 0.3 to $0.7 \mathrm{pc}$ for the clusters with known distances.

In the $2 \mathrm{CD}$ analysis, it is fundamental to decontaminate the clusters for field stars. The points shown in the diagrams are the probable members resulting from a statistical realisation for each cluster. In Fig. 7 we present the 2CD for DBSB 011, to illustrate the method. The left panel shows the surrounding field stars (offcluster) and the right panel shows the assumed cluster area (oncluster), with a symbol size proportional to $P_{\text {jon }}$.

Figures 8 and 9 display the decontaminated $((J-H)$, $\left.\left(H-K_{\mathrm{s}}\right)\right)$ diagram, which shows intrinsic colours of main sequence (MS) stars from O3 to M5 (see Binney \& Merrifield 1998, and references therein). Reddening lines with a slope $E(J-H) / E\left(H-K_{\mathrm{s}}\right)=1.72$ (Schlegel et al. 1998) for an O3 MS star and an M5 giant, as well as the locus of unreddened T Tauri stars (Meyer et al. 1997) are indicated.

The source distribution along the $2 \mathrm{CD}$ is typical of embedded clusters for most objects in the sample, i.e., an important reddening is observed and sources can be seen in the diagram region which T Tauri and AeBe stars occupy.

The number of members derived from the statistical method for each cluster was compared to its Ic index. The correlation coefficient is $r=0.96$. About $40 \%$ of the sources are not detected in all three bands and were not considered in the $2 \mathrm{CD}$ analysis, although they were included in the $I c$ index calculation. Therefore, this index points to a larger number of members than those obtained from the $2 \mathrm{CD}$ analysis.

Only a few probable members in the decontaminated 2CD can be found in objects DBSB 030, DBSB 039, DBSB 042, 


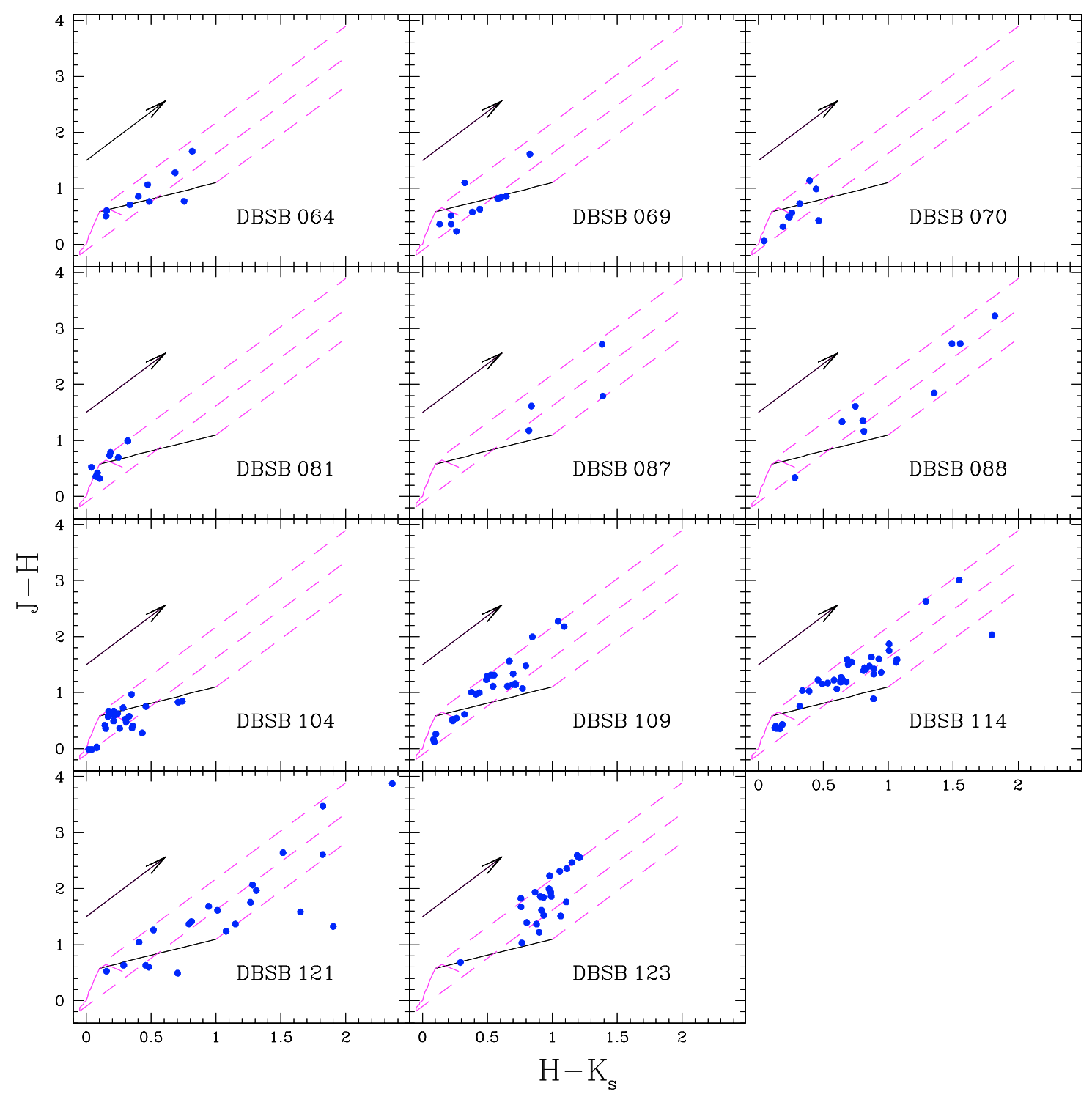

Fig. 9. $\left((J-H),\left(H-K_{\mathrm{s}}\right)\right)$ diagrams for the remaining objects. Symbols as in Fig. 8.

DBSB 081 and DBSB 087. These results, however, are not statistically significant so that deeper observations are recommended.

Interstellar extinction of the stars located in the domain of normal reddening in the $\left((J-H),\left(H-K_{\mathrm{s}}\right)\right)$ diagram, containing weak T Tauri stars, and in the domain of classical $K_{\mathrm{s}}$ values can be obtained by using reddening vectors and intrinsic colours (Soares et al. 2005).

The $A_{J}$ mean absorption for the clusters in Table 1 indicates that they are not deeply embedded. The extinction values for the stars with available spectroscopy is lower than the mean value of their respective clusters, owing to differential reddening.

Figure 10 shows the $K_{\mathrm{s}}$ excess fraction histogram of the embedded clusters in the sample, except for the low statistic clusters above. Most of the clusters present a $K_{\mathrm{s}}$ excess fraction in the range $0.1-0.3$ (Table 1), and none exhibits more than $50 \%$ of stars with a $K_{\mathrm{s}}$ excess in the sample. Typical errors in $K_{\mathrm{s}}$ fractions are 0.05 .

By means of the percentage of $K_{\mathrm{s}}$ excess stars, one can estimate the age of the cluster or stellar group. Most of the clusters present about $20 \%$ of the PMS stars with infrared excess, which suggests an evolutionary stage similar to that of the IC 348 cluster which is 2-3 Myr old (Haisch et al. 2001). In the sample,
Table 3. Age and age dispersion of the clusters based on PMS fits.

\begin{tabular}{ccc}
\hline \hline Cluster & Age (Myr) & Age dispersion (Myr) \\
\hline DBSB 031 & $0.9 \pm 0.3$ & $1.6 \pm 0.7$ \\
DBSB 049 & $2.1 \pm 0.3$ & $2.9 \pm 0.4$ \\
DBSB 069 & $3.0 \pm 1.1$ & $3.1 \pm 1.7$ \\
DBSB 088 & $1.2 \pm 0.5$ & $1.6 \pm 0.9$ \\
DBSB 104 & $6.0 \pm 0.9$ & $3.5 \pm 0.4$ \\
DBSB 109 & $1.1 \pm 0.4$ & $2.0 \pm 0.8$ \\
\hline
\end{tabular}

5 clusters have a $K_{\mathrm{s}}$ excess fraction larger than $40 \%$, as is the case of the Taurus dark clouds, which have an estimated age of about 1-2 Myr (Kenyon \& Hartmann 1995).

The age determination from the PMS isochrone fit was applied only to the clusters with known distance. Table 3 shows the age and age dispersion for each cluster obtained from the statistical realisations, together with their respective errors.

There may be residual field contamination in the cluster $2 \mathrm{CDs}$. The age dispersions are not only non-negligible but also compatible with the expected dispersions for the cluster formation stage. The dispersions were obtained from the values 


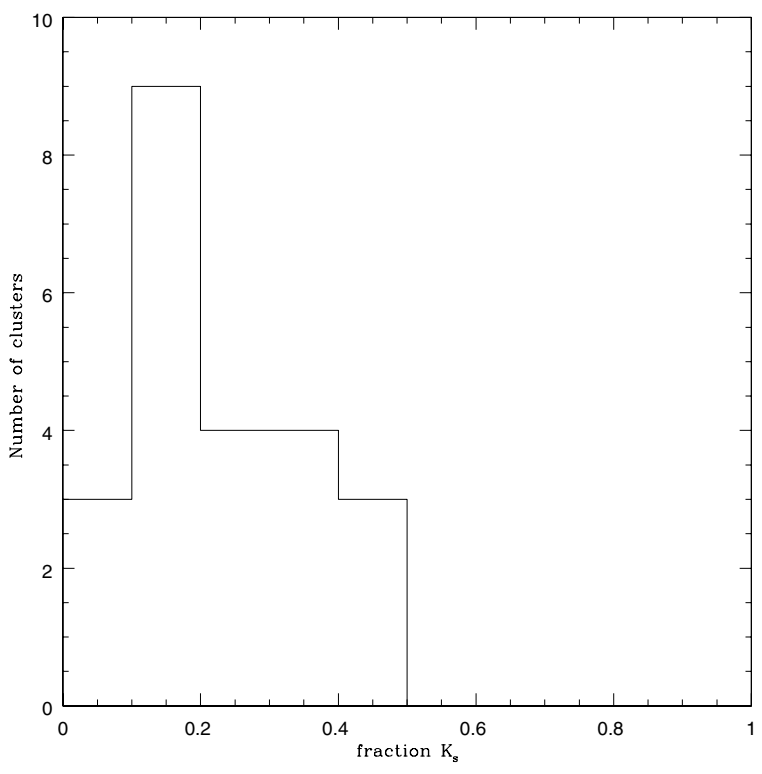

Fig. 10. $K_{\mathrm{s}}$-excess fraction histogram of the clusters in the sample with good statistics.

found from several realisations of the statistical subtraction (Sect. 4). The error presented in Table 3 does not include the reddening and distance estimation uncertainties. It is probable that due to these errors, there is not a precise correlation between the $K_{\mathrm{s}}$ fraction and the age derived from the PMS method (Table 3 ).

The total mass estimated for each cluster with known distance was obtained assuming a universal IMF (Sect. 4). We used isochrones of 1 and $2 \mathrm{Myr}$, according to the evolutionary PMS stage of each cluster in this sample. The derived total mass for most clusters turned out to be lower than $M_{\mathrm{T}}=100 M_{\odot}$ (Table 1).

The masses estimated for the clusters in the sample indicate them to be unbounded stellar systems (Kroupa \& Boily 2002)

\section{Concluding remarks}

A sample of 22 candidate embedded stellar systems in reflection nebulae and/or HII environments were investigated by means of optical spectroscopy and near infrared photometry. Our analysis was based on source surface density and 2CDs, using 2MASS data together with theoretical PMS isochrones.

These stellar systems appear to be low mass embedded stellar clusters containing few stars. Their $A_{J}$ absorptions and ages point to an evolutionary stage similar to that of the clusters in the reflection nebulae studied in Soares et al. (2005).

In the sample of clusters with the available spectroscopic data gathered at CASLEO, DBSD 049 contains the most massive member, a B0V star. Therefore, the clusters in this sample including those in an HII environment, were formed without the presence of a massive star ealier than B-type.

The total mass estimated for each cluster with known distance was obtained assuming a universal IMF. The derived total mass for most clusters turned out to be lower than $M_{\mathrm{T}}=100 M_{\odot}$.
This fact indicates that they are unbound stellar systems according to Kroupa \& Boily (2002). Apparently, embedded low mass clusters have been supplying stars to the Galactic field for generations (Soares et al. 2005).

Acknowledgements. We would like to thank the CASLEO staff members and technicians for their kind hospitality and support during the observing runs. We are grateful for the use of the CCD and data acquisition system at CASLEO, supported under US National Science Foundation (NSF) grant AST-90-15827. We also thank the anonymous referee for his/her helpful remarks. This publication makes use of data from the Two Micron All Sky Survey, which is a joint project of the University of Massachusetts and the Infrared Processing and Analysis Center, founded by the National Aeronautics and Space Administration and the NSF. We employed data from the CDS database (Strasbourg). We thank the financial support from the Brazilian institution CNPq. J.B.S. is especially thankful for the CNPq fellowship he was awarded. This work was also partially supported by the Argentinian institutions CONICET and SECYT (Universidad Nacional de Córdoba).

\section{References}

Ahumada, A. V., Clariá, J. J., Bica, E., \& Piatti, A. E. 2000, A\&A, 141, 79 Ahumada, A. V., Clariá, J. J., Bica, E., Dutra, C. M., \& Torres, M. C. 2001, A\&A, 377, 845

Bica, E., Dutra, C. M., \& Barbuy, B. 2003a, A\&A, 397, 177

Bica, E., Dutra, C. M., Soares, J., \& Barbuy, B. 2003b, A\&A, 404, 223

Binney, J., \& Merrifield, M. 1998, Galactic Astronomy, ed. J. P. Ostriker, \& D. N. Spergel, Princeton, 107

Brand, J., Blitz, L., \& Wouterloot, J. G. A. 1986, A\&AS, 65, 537

Caswell, J. L., \& Haynes, R. F. 1987, A\&A, 171, 261

Carpenter, J. M., Snell, R. L., Schloerb, F. P., \& Skrutskie, M. F. 1993, ApJ, 407, 657

D’Antona, F., \& Mazzitelli, I. 1997, Evolution of low mass stars in cool stars in Clusters and Associations, ed. G. Micela, \& R. Pallavicini, Mem. S. A. It., 68,807

D’Antona, F., \& Mazzitelli, I. 1998, A role for superadiabatic convection in low mass structures, in Brown Dwarfs and Extrasolar Planets, ed. R. Rebolo, E. Martin, \& M. R. Zapatero Osorio, ASP Conf. Ser., 442

Dutra, C. M., Bica, E., Soares, J., \& Barbuy, B. 2003, A\&A, 400, 533

Gum, C. S. 1955, Mem. RAS, 67, 15

Haisch, K. E., Jr., Lada, E. A., \& Lada, C. J. 2001, AJ, 121, 2065

Ivanov, V. D., Borissova, J., Pessev, P., Ivanov, G. R., \& Kurtev, R. 2002, A\&A, 394, L1

Kenyon, S. J., \& Hartmann, L. 1995, ApJS, 101, 117

Kroupa, P., \& Boily, C. M. 2002, MNRAS, 336, 1188

Kuchar, T. A., \& Clark, F. 1997, ApJ, 488, 224

Lada, C. J., \& Adams, F. 1992, ApJ, 393, 278

Lada, C. J., \& Lada, E. A. 2003, ARA\&A, 41, 115

Lauberts, A. 1982, ESO/Uppsala survey of the ESO(B) atlas, Garching: ESO

Mercer, E. P., Clemens, D. P., Meade, M. R., et al. 2005, ApJ, 635, 560

Meyer, R. M., Calvet, N., \& Hillenbrand, L. A. 1997, AJ, 114, 288

Muench, A. A., Lada, E. A., Lada, C. J., \& Alves, J. 2002, ApJ, 573, 366

Rodgers, A. W., Campbell, C. T., \& Whiteoak, J. B. 1960, MNRAS, 121, 103

Schlegel, D. J., Finkbeiner, D. P., \& Davis, M. 1998, ApJ, 500, 525

Schmidt-Kaler, T. 1982, in Landolt-Börnstein, New Series, Group VI, Vol. 2b (Springer-Verlag, Berlin), 1

Silva, D. R., \& Cornell, M. E. 1992, ApJS, 81, 865

Skrutskie, M., Schneider, S. E., Stiening, R., et al. 2006, AJ, 131, 1163

Soares, J. B. 2006, Estágios iniciais de aglomerados estelares, Ph.D. Thesis, UFRGS, Porto Alegre, Brazil

Soares, J. B., \& Bica, E. 2002, A\&A, 388, 172

Soares, J. B., \& Bica, E. 2003, A\&A, 404, 217

Soares, J. B., Bica, E., Ahumada, A. V., \& Clariá, J. J. 2005, A\&A, 430, 987

Stone, R. P. S., \& Baldwin, J. A. 1983, MNRAS, 204, 347

Testi, L., Palla, F., \& Natta, A. 1998, A\&AS, 133, 81 